\title{
The invasion and coexistence of competing Wolbachia strains
}

\author{
MJ Keeling ${ }^{1,2}$, FM Jiggins ${ }^{3}$ and JM Read ${ }^{1,2}$ \\ ${ }^{1}$ Mathematics Institute, University of Warwick, Gibbet Hill Rd., Coventry CV4 7AL, UK; ${ }^{2}$ Department of Biological Sciences, University \\ of Warwick, Gibbet Hill Rd., Coventry CV4 7AL, UK; ${ }^{3}$ Department of Genetics, University of Cambridge, Downing Street, Cambridge \\ CB2 3EH, UK
}

\begin{abstract}
Cytoplasmic incompatibility between arthropods infected with different strains of Wolbachia has been proposed as an important mechanism for speciation. However, a basic requirement for this mechanism is the coexistence of different strains in neighbouring populations. Here we test whether this required coexistence is possible in a spatial context. Continuous-time models for the behaviour of one and two strains of Wolbachia within a single well-mixed
\end{abstract}

population demonstrate the Allee effect and founder control, such that one strain is always driven extinct. In contrast, discretised spatial models show patchy persistence of the two strains although coexistence within the same habitat is rare. A simplified model of such founder control suggests that it is fragmentation of (or barriers within) the habitat rather than space itself that leads to persistence.

Heredity (2003) 91, 382-388. doi:10.1038/sj.hdy.6800343

Keywords: Wolbachia; cytoplasmic incompatibility; speciation; spatial structure

\section{Introduction}

Wolbachia is a bacterial symbiont found in all the major arthropod groups and filarial nematodes. It is an alphaproteobacterium that lives in the cell cytoplasm of its host, and is transmitted through the egg from mother to offspring. It has been estimated that $16-22 \%$ of insects and $26 \%$ of crustacean species are infected with Wolbachia (Werren et al, 1995; Bouchon et al, 1998; West et al, 1998), and some studies have suggested that these figures may substantially underestimate their true incidence (Jeyaprakash and Hoy, 2000; Jiggins et al, 2001).

In insects and crustaceans, many Wolbachia strains cause cytoplasmic incompatibility (CI) (Laven, 1951; Yen and Barr, 1971; O'Neill et al, 1992). In diploid hosts, this results in zygotic death of potential offspring when an infected male is crossed with an uninfected female. However, all other crosses are unaffected. CI therefore allows the bacterium to spread through the population by reducing the fitness of uninfected females in the population (Caspari and Watson, 1959), and such spreads have been observed in natural populations (Turelli and Hoffmann, 1991).

Different strains of Wolbachia may be mutually (bidirectionally) incompatible (Breeuwer and Werren, 1990; O'Neill and Karr, 1990). That is, if a male infected with one strain is crossed to a female infected with a second strain, inviable zygotes result. Therefore, if two populations are infected with different strains, then crosses between them will be incompatible. The potential importance of this as a speciation mechanism was immediately recognised: if two different populations were bidirectionally incompatible, then gene flow between them is dramatically reduced or eliminated

Correspondence: M Keeling, Department of Biological Sciences \& Maths Institute, University of Warwick, Gibbet Hill Rd., Coventry CV4 7AL, UK. E-mail: m.j.keeling@warwick.ac.uk

Received: 14 March 2003 entirely (Laven, 1959; Werren, 1998). It is clear that Wolbachia does not cause most of the observed cases of reproductive isolation in insects, which tend to initially involve sterility or inviability of the heterogemetic sex (Coyne and Orr, 1989). However, this does not preclude the possibility that Wolbachia can sometimes play a role in speciation events.

If Wolbachia does play an important role in speciation, then we expect to find Wolbachia-induced incompatibilities between populations and pairs of sibling species. Several studies have found this to be the case, although none have produced conclusive evidence that Wolbachia causes speciation. In the sympatric jewel wasps, Nasonia vitripennis and N. giraulti, Wolbachia cause F1 hybrid inviability. However, it is unclear whether Wolbachia itself played a role in speciation, as there is also premating isolation and F2 hybrid breakdown (Breeuwer and Werren, 1990). In a second pair of jewel wasps, $N$. longicornis and $N$. giraulti, CI is the only cause of postmating reproductive isolation, but in nature these species are allopatric, and therefore isolated by geography (Bordenstein et al, 2001). Other studies have found evidence for bidirectional CI between populations of the same species. The best-characterised system is Drosophila simulans, where several bidirectionally incompatible Wolbachia types exist in different populations ( ${ }^{\prime}$ Neill and Karr, 1990; Montchamp-Moreau et al, 1991; Merçot et al, 1995). More complex patterns of bidirectional and unidirectional incompatibility exist between populations (or sibling species) of the mosquito Culex pipiens (Hoffmann and Turelli, 1997). In some instances, strong incompatibilities were found between insects collected from within a few kilometres of each other or from the same locality (Barr, 1980; Magnin et al, 1987). However, it is not clear whether this is caused by different Wolbachia strains being maintained in adjacent populations.

Therefore, empirical studies suggest there may be many cases where different host populations harbour different strains of Wolbachia. Theoretical studies suggest 
that this can substantially increase the level of genetic divergence between populations in the face of migration (Telschow et al, 2002a, b).

Despite Wolbachia-induced incompatibilities being widespread in nature, it has been argued that they may be unlikely to be important agents causing speciation (Hurst and Schilthuizen, 1998; Shoemaker et al, 1999; Weeks et al, 2002). One criticism originates from theoretical studies that have shown that within a randomly mating population different Wolbachia strains are not expected to coexist (Caspari and Watson, 1959; Rousset et al, 1991). Therefore, it was argued that it was unlikely that different Wolbachia strains would be able to coexist in neighbouring populations (Shoemaker et al, 1999). The aim of this paper is to test this assertion by examining the conditions under which stable contact zones can be formed between bidirectionally incompatible CI types in adjoining populations.

Previous models have considered the dynamics of a single CI type in a spatially structured population (Turelli and Hoffmann, 1991; Wade and Stevens, 1994; Schofield, 2002) and multiple strains in a panmictic population (Rousset et al, 1991). However, the dynamics of multiple strains within a structured population have only been considered verbally before (Hoffmann and Turelli, 1997).

\section{Single strain model}

The models developed throughout this paper assume that Wolbachia is only transmitted vertically; that is, it can only be passed from mother to offspring. The transmission of infection from mother to offspring is not perfect however, and occurs with probability $\tau$.

Initially, we subdivide the population into four classes, uninfected females, $F_{U}$, infected females, $F_{I}$, uninfected males, $M_{U}$, and infected males, $M_{I}$. These compete through the action of a density-dependent death rate, such that in the absence of infection the population obeys a logistic growth equation. When an infected male is crossed with an uninfected female - or more precisely when an infected male fertilizes an uninfected egg zygotic death due to cytoplasmic incompatibility is assumed to occur (no offspring are produced) with probability $q$. Although CI gives a benefit to infected females through increased mating success, for generality we assume that those individuals infected have slightly lower natural fitness; as such their density-dependent death rate is increased by an amount $D$. For simplicity we shall often quote results and show simulations for the case when $D=0$, as this does not change the qualitative conclusions. Finally, we assume continuous time dynamics with overlapping generations; the alternative (discrete time and nonoverlapping generations) has also been simulated and produces qualitatively similar conclusions. The governing equations for the four classes are:

$$
\begin{aligned}
\frac{\mathrm{d} F_{I}}{\mathrm{~d} t} & =f \tau b F_{I}-(d+D) T F_{I} \\
\frac{\mathrm{d} F_{U}}{\mathrm{~d} t} & =f b\left(F_{U}+(1-\tau) F_{I}\right)\left[1-q \frac{M_{I}}{M_{U}+M_{I}}\right]-d T F_{U} \\
\frac{\mathrm{d} M_{I}}{\mathrm{~d} t} & =m \tau b F_{I}-(d+D) T M_{I} \\
\frac{\mathrm{d} M_{U}}{\mathrm{~d} t} & =m b\left(F_{U}+(1-\tau) F_{I}\right)\left[1-q \frac{M_{I}}{M_{U}+M_{I}}\right]-d T M_{U}
\end{aligned}
$$

where $T$ is the total population size, $b$ is the natural birth rate, $f(=1-m)$ is the proportion of individuals born female and $d$ is the density-dependent death rate for uninfected individuals.

While this set of equations obviously allows the simulation of the Wolbachia dynamics, such a system is too complex to allow clear analytical insights. We notice however, that after one or two generations, the ratio of males to females in each state is identical, $M_{U}: F_{U}=M_{I}: F_{I}$. This vastly simplifies the system, and we can rewrite the equations solely considering the entire infected $(I)$ and uninfected $(U)$ populations. With suitable parameter rescaling:

$$
\begin{aligned}
& \frac{\mathrm{d} I}{\mathrm{~d} t}=\tau b I-(d+D) T I \\
& \frac{\mathrm{d} U}{\mathrm{~d} t}=b(U+(1-\tau) I)\left[1-q \frac{I}{T}\right]-d T U
\end{aligned}
$$

Even this reduced set of two coupled differential equations is not trivial to understand, therefore we examine the proportion of the population that is infected, $\xi=I /(I+U)$ :

$$
\frac{\mathrm{d} \xi}{\mathrm{d} t}=-b \xi(1-\tau)-D T \xi(1-\xi)+b q(1-\tau \xi) \xi^{2}
$$

This differential equation clearly demonstrates that there is an Allee effect (Courchamp et al, 1999), such that a small level of infection cannot invade an uninfected population (when $\xi$ is sufficiently small, its rate of change is negative; hence the level of infection decreases). It is only when the level of infection breaks some critical threshold that the infection takes off (Caspari and Watson, 1959). This corresponds exactly with the Allee effect where low-density populations are driven to extinction, but high-density populations can survive. Looking at the simplified case where $D=0$, we find that:

$$
\xi_{\text {threshold }}=\frac{1-\sqrt{1-4 \chi}}{2 \tau} \quad \xi_{\text {equilibrium }}=\frac{1+\sqrt{1-4 \chi}}{2 \tau}
$$

where $\chi=(1-\tau) \tau / q$. Hence, if $\tau$ or $q$ is too small, infection cannot persist in the population.

This Allee effect raises the interesting question of how Wolbachia could invade a totally susceptible population. Three distinct possibilities exist. Firstly, $\tau$ is so close to one that the threshold is practically zero; therefore invasion can occur from a very low density of infected organisms. Secondly, the disease invades when the total or local subdivided population size is small, so that even with just a few infectious individuals the population is above the threshold. Finally, there could be sufficient immigration of infection from an external source to counteract the Allee effect. Which of these is the most likely scenario will depend on the precise population dynamics and effects of Wolbachia.

\section{Two strain models}

Using the basic framework developed above, we can consider the motivating question of competition between two (or more) strains of Wolbachia, and whether coexistence is possible. If only maternal transmission can occur, then the two strains must remain isolated. We can therefore model the numbers of three distinct types of 
organism, those uninfected, $U$, those infected with one strain, I, and those infected with the other, J. For generality, the two strains are considered to have different parameters:

$$
\begin{aligned}
\frac{\mathrm{d} I}{\mathrm{~d} t} & =\tau_{I} b I\left[1-q_{J} \frac{J}{T}\right]-\left(d+D_{I}\right) T I=f_{I}(I, J, U) \\
\frac{\mathrm{d} J}{\mathrm{~d} t} & =\tau_{J} b J\left[1-q_{I} \frac{I}{T}\right]-\left(d+D_{J}\right) T J=f_{J}(I, J, U) \\
\frac{\mathrm{d} U}{\mathrm{~d} t} & =b\left(U+\left(1-\tau_{I}\right) I+\left(1-\tau_{J}\right) J\right)\left[1-q_{I} \frac{I}{T}-q_{J} \frac{J}{T}\right]-d T U \\
& =f_{U}(I, J, U)
\end{aligned}
$$

To simplify the notation in what follows, we have introduced three functions, $f_{I}, f_{J}$ and $f_{U}$, which are the rates of changes of the three populations.

From this model, it is clear that when the system is at the single strain equilibrium (such that $\left.f_{I}\left(I^{*}, 0, U^{*}\right)=f_{U}\left(I^{*}, 0, U^{*}\right)=0\right)$, then a small amount of the other strain $J$ cannot invade as it always performs worse than the uninfected population $\left(f_{J}\left(I^{*}, J, U\right)<f_{U}\left(I^{*}, J, U^{*}\right) \approx 0\right.$ $J \ll 1)$ - although both uninfected and strain $J$ both suffer from cytoplasmic incompatibility with the dominant $I$ strain, strain $J$ fairs worse as its vertical transmission is not guaranteed. The two strains therefore exhibit founder control due to this competitive exclusion - neither strain can invade from low levels when the other strain is already present. We also note that there is no stable equilibrium solution where the two strains $I$ and $J$ coexist (Rousset et al, 1991). Hence, in a homogeneously mixed population, one strain must always dominate; which strain this is depends on the particular parameters associated with each strain and their initial densities (Rousset et al, 1991).

Due to the form of indirect competition between the two strains, almost any form of aggregate (homogeneous) model will predict founder control. However, multiple strains clearly exist in the natural world; therefore the question arises as to what factors can account for this persistence. As with many model systems, spatial heterogeneity is a plausible candidate to explain the increased coexistence (Slatkin, 1974; Hanski, 1983; Tilman, 1994; Bolker and Grenfell, 1995; Pacala and Levin, 1996). This could be induced by underlying environmental heterogeneities such that different regions favour different strains - although this is difficult to envisage. A more appealing concept is that spatial heterogeneity arises naturally from the subdivision of the population and the dynamics within each subpopulation. This may be intuitively considered a partial geographic barrier to gene flow, such that the mixing within a habitat (subpopulation) is much greater than the mixing between them.

\section{A spatial model}

We can make the model system spatial by subdividing the landscape into a set of habitats, where the population in habitat $i$ is denoted by the appropriate subscripts. Small amounts of movement are allowed between habitats, leading to the following equations:

$$
\frac{\mathrm{d} I_{i}}{\mathrm{~d} t}=f_{I}\left(I_{i}, J_{i}, U_{i}\right)+\mu\left[\sum_{j} \sigma_{i j} I_{j}-I_{i} \sum_{j} \sigma_{j i}\right]
$$

$$
\begin{aligned}
& \frac{\mathrm{d} J_{i}}{\mathrm{~d} t}=f_{J}\left(I_{i}, J_{i}, U_{i}\right)+\mu\left[\sum_{j} \sigma_{i j} J_{j}-J_{i} \sum_{j} \sigma_{j i}\right] \\
& \frac{\mathrm{d} U_{i}}{\mathrm{~d} t}=f_{U}\left(I_{i}, J_{i}, U_{i}\right)+\mu\left[\sum_{j} \sigma_{i j} U_{j}-U_{i} \sum_{j} \sigma_{j i}\right]
\end{aligned}
$$

where $\sigma$ (equal to 0 or 1 ) defines the allowed movements, and $\mu$ determines their magnitude. We define the level of coupling between populations as $\mu \sum_{j} \sigma_{i j}$, which is the rate at which individuals disperse out of a patch. This is a very general formulation and can accommodate a wide variety of spatial configurations. In what follows, we discretise the entire space into a grid of equal-sized habitats, and focus primarily on when movement is only between adjacent habitats - this is an example of a locally coupled lattice model (Hassell et al, 1991; Keeling, 1999). We contrast this locally coupled model with global movement between habitats.

Figure 1a shows an example of the typical spatial configuration, starting with a random distribution of strains, which results from small levels of local coupling. This spatial pattern is stable and arises due to the dynamic enhancement of small heterogeneities in the initial configuration. We clearly see coexisting patches of infection dominated by one or other of the two strains. Therefore, in moving from a homogeneous to a spatial system, we have enabled the two strains to coexist, although at each location one strain generally dominates and the other is forced to extinction. Only in the boundaries between patches can local coexistence be maintained by the influx of both strains. This simple concept can be generalised to allow multiple strains to coexist in a patchy manner.

Although the two-dimensional lattice implemented in Figure 1a is the most biologically natural approach, it is often more intuitive (and computationally feasible) to consider a one-dimensional line of habitats, again with local movement. Figure $1 \mathrm{~b}$ shows the development of a stationary spatial pattern in a one-dimensional system of habitats. Starting with a random distribution of strains, regions dominated by strain $I$ (black) or strain $J$ (grey) soon emerge. We note that due to the parameters used in this simulation narrow patches are not viable and are 'devoured' by the surrounding strain.

For a given set of infection parameters, the level of localized movement, $\mu$, determines the width of habitat that can survive. We assume that strain $I$ is more fit than strain $J\left(\tau_{I}>\tau_{J}\right.$ and $\left.q_{I}>q_{J}\right)$, and look for the critical value of $\mu$ at which a small patch of strain $J$ can survive (Figure 2a, solid line). Simulations are started with a small number of contiguous habitats at the $J$ strain equilibrium, while all other habitats are at the $I$ strain equilibrium. Clearly, large patches are able to withstand higher movement rates, but the asymptote is soon reached. For the same disease parameters, we also consider the movement rate necessary for strain $J$ to invade an uninfected population (dashed line). In the region between these two curves, strain $J$ can coexist spatially with strain $I$, but equally important strain $J$ (and also strain $I$ ) can colonise new uninfected habitat. This graph should be compared to Figure $2 b$, which shows similar results for a population with global movement; in this scenario it is the total amount of each strain present in the population that determines the long-term 
dynamics. Simulations are begun with habitats in either the $I$ strain or $J$ strain equilibrium. These two graphs (Figure 2a and $b$ ) represent two extremes of movement; reality is likely to lie somewhere in between.

For the local movement scenario, Figure 2c considers this movement threshold for different sets of strain $J$ parameters. Below the black surface, a single habitat of $J$ can survive in a sea of strain $I$, and similarly below the grey surface a single $I$ habitat can survive in a sea of $J$. Therefore, if the movement level is below both curves, a patchy distribution can be maintained. We note that when the transmission $\left(\tau_{J}\right)$ and cytoplasmic incompat-

a

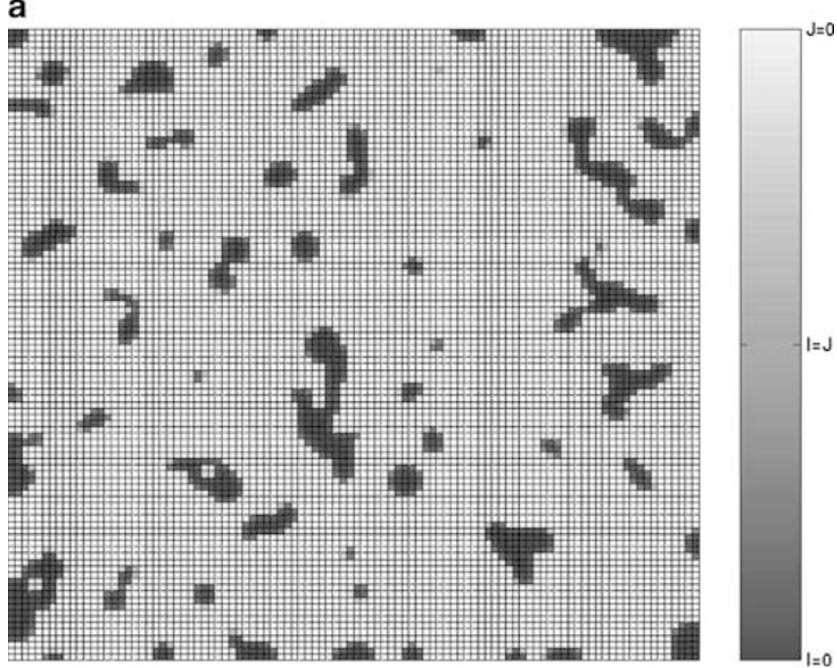

b
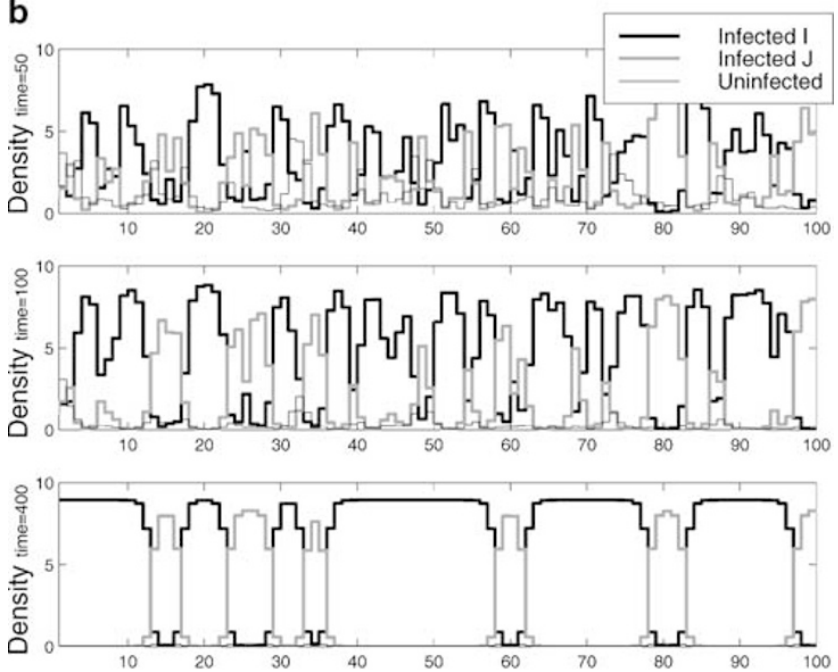

Figure 1 Graph (a) is an example of the typical steady spatial configurations of a two-dimensional arrangement of habitats. The black areas show dominance by strain $I\left(\tau_{I}=0.9, q_{I}=0.96, D_{I}=0\right)$, the white areas are dominated by strain $J\left(\tau_{J}=0.85, q_{J}=0.92, D_{I}=0\right)$ and grey areas are regions of coexistence. We have assumed coupling of strength $\mu=0.004$ to the nearest four neighbours, and birth and death rates of $b=0.1$ and $d=0.01$. Graph (b) shows the time evolution to the steady state in a one-dimensional system, giving the density of strain $I$ (thick black line), strain $J$ (thick grey line) and uninfected individuals (thin black line). Initially, the population levels in each patch are randomly chosen. The parameters are $\tau_{I}=0.9, q_{I}=0.96, D_{I}=0, \tau_{I}=0.85, q_{I}=0.92, D_{I}=0$, $\mu=0.008, b=0.1$ and $d=0.01$. ibility $\left(q_{J}\right)$ parameters are low; those infected with strain $J$ cannot out-compete the uninfected individuals; so strain $J$ is doomed to rapid extinction. This extinction occurs when $\chi$ (given above) is greater than $1 / 4$.

\section{Continuous spatial models}

The spatial model given above, which demonstrates the persistence of the two species, contains the implicit assumption that within each habitat there is complete random mixing, but between neighbouring habitats the level of mixing is small - there are some form of geographic barriers to spread (Keeling, 1999). We are therefore examining the dynamics at a large spatial scale. If the environment is not broken into discrete patches, but the habitat is assumed to be large and continuous, then this patchy model formulation is flawed. To investigate this situation, we consider an idealised stochastic spatial model, where individuals are distributed in space and interact with a set of nearby individuals. To simplify the behaviour, we ignored the true behaviour of Wolbachia infections and concentrated on the emergent phenomenon of founder control.

Individuals within this model can be in one of two states, which for conformity we shall call $I$ and $J$. The fecundity of the two strains is determined by their local neighbourhood, such that fecundity increases with the proportion of neighbours of the same strain. This clearly produces local dynamics that display founder control, as an isolated $I$ in a sea of $J$ (and vice versa) would have a very low reproductive success. This simple model of founder control consistently predicts the inevitable extinction of the weaker strain, and this is largely independent of the initial conditions. Figure 3 shows that when there are just two large patches, the stronger strain always invades in a wave-like manner with the invasion front moving with a constant velocity. This result is qualitatively robust to a wide range of changes in the model structure, parameters and spatial interactions.

\section{Discussion}

It is now well documented that when individuals from different populations are crossed, Wolbachia-induced cytoplasmic incompatibility can cause the zygotic death of hybrid offspring (reviewed in Werren, 1997). However, if this is to lead to the speciation of those populations, it is necessary that bidirectionally incompatible Wolbachia infections can form stable contact zones between populations. We have found that this can occur where the habitat is patchy and fragmented, but not when the population is continuous and uniform.

The continuous time model of a single Wolbachia strain within a randomly mating population shows an Allee effect, such that invasion of the bacterium is not possible until infected individuals exceed a density threshold (Caspari and Watson, 1959; Fine, 1978). Therefore, the initial invasion of the bacterium requires either that the infection arises within a small or structured population, that there are high enough rates of immigration to exceed the threshold or that the parameters are such that the threshold is extremely small. 
The model of two bidirectionally incompatible Wolbachia strains shows that long-term coexistence is not generally possible within the same well-mixed population, which is again in agreement with earlier work (Caspari and Watson, 1959). If two infections do arise within a population, then there is strong founder control. Therefore, everything else being equal, the commoner of the two infection types will persist, while the rarer will go extinct.
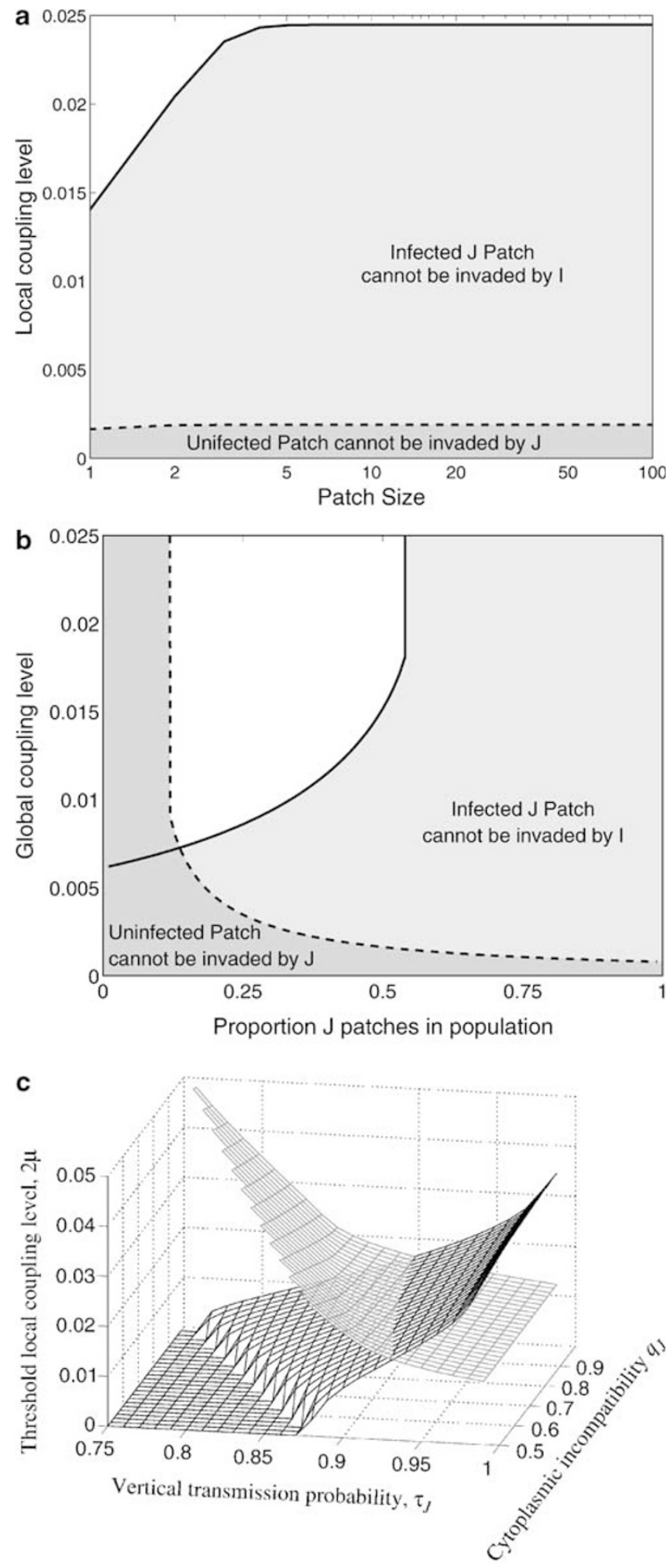

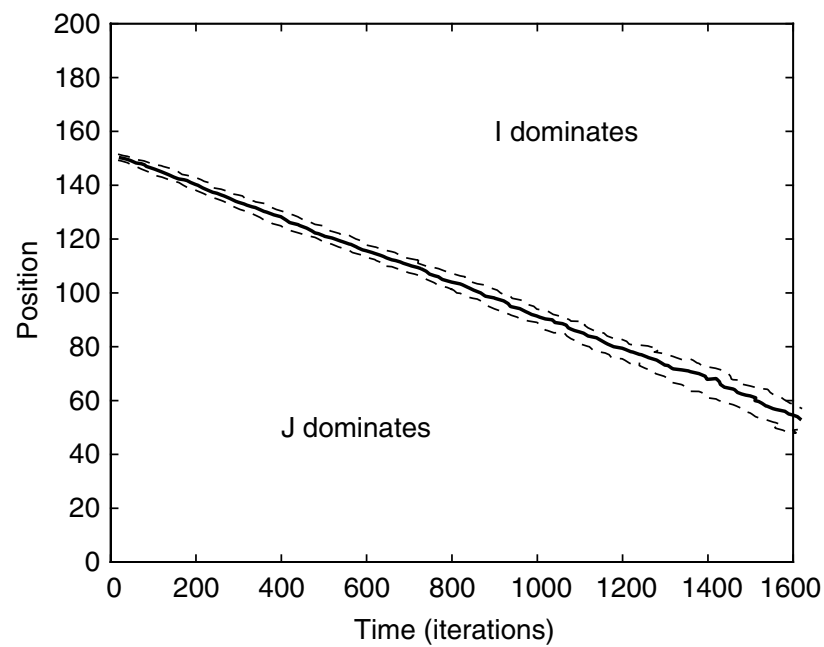

Figure 3 Results from the individual-based model (a cellular automaton) with local founder control performed on a regular two-dimensional lattice with four nearest neighbours. Starting from two large patches of single strains at equilibrium, the lines show the average spatial location where strain $I$ is at 25,50 and $75 \%$ of its carrying capacity. This clearly shows that in a homogeneous spatial system, one strain dominates and spreads in a wave-like manner; for this parameter set the wave speed is approximately one cell every 16.5 interactions (death rate $I=0.15$, death rate $J=0.2$, reproductive rate of $I=0.2^{*}$ empty sites $+1.0^{*} I$ sites in the neighbourhood, reproductive rate of $J=0.1^{*}$ empty sites $+1.0^{*} J$ sites in the neighbourhood).

Spatial structure has long been known to promote the coexistence of competitors (Tilman, 1994; Pacala and Levin, 1996); here we investigate its effect on two strains of Wolbachia. First, we considered a grid of randomly mixing populations, with neighbours connected by migration. Under these conditions, a single strain dominates within each localised subpopulation, but different strains can persist in neighbouring subpopulations. This can be viewed as an example of the small parameter model of Karlin and McGregor (1972). The stability of a given patch of one strain depends mainly on the local parameters but in part on the size of the patch; if it is too small, the relatively high level of immigration from neighbouring patches may allow invasion of the other strain, a phenomenon known as patch swamping (Hanson, 1966).

In an attempt to understand the role played by different forms on insect dispersal, we contrasted the results of local movement, with those of global movement, where insects are free to disperse to any available

Figure 2 Persistence and invasion thresholds for the one-dimensional model. Graph (a): starting from a patch of strain $J$ (at single strain equilibrium) of a given width, two critical levels of local coupling are shown. First, the maximal level of coupling that allows strain $J$ to persist in a sea of strain I (solid curve). Second, the minimal level of coupling that allows strain $I$ to invade a sea of uninfected individuals (dashed curve). For the one-dimensional model with local movement, the level of dispersal or coupling is $2 \mu$. The same parameters are used as in Figure 1b. Graph (b) shows the same invasion curves for global movement of individuals; here the level of coupling is given by $N \mu$, where $N$ is the total number of habitats. For the model with local movement, graph (c) shows how the critical level of coupling for persistence of a single patch varies with the parameters for strain $J\left(\tau_{I}=0.85, q_{I}=0.92\right.$ and $\left.D_{I}=D_{J}=0\right)$. 
habitat. Here it is the initial proportion of habitats that are at the $J$ strain equilibrium (rather than patch size) that determines the invasion thresholds. For local dispersal, even very small patches can be stable or even colonise the surrounding habitat. When the coupling is global however, we effectively see founder control at the habitat level, where the initial proportion of habitat types is crucial in determining the long-term behaviour. In principle, the actual dispersal on insects is likely to be dominated by local movement, although some longerrange dispersal will take place. Invasion of Wolbachia or a new strain is therefore likely to proceed by a mixture of long-range jumps and irregular localised spread (Turelli and Hoffmann, 1995).

The second spatial model we investigated was at a more local scale, simulating individuals in a continuous, homogenous habitat. Following a reductionist principle, this model ignored the detailed dynamics of Wolbachia and focused instead on the emergent property of founder control. The outcome in this situation is quite different, with the strain that has the higher transmission efficiency or causes the greater level of incompatibility spreading as an advancing wave. After testing a variety of situations, we firmly believe that this result is robust for any form of founder control in an individual-based system with local interactions and is not dependent on the model assumptions. We therefore conclude that spatial heterogeneity in migration rates (the difference between within-patch and between-patch interaction) is necessary for bidirectionally incompatible Wolbachia types to stably coexist in neighbouring populations.

This behaviour is strikingly similar to that of hybrid zones, which occur when incompatibilities between nuclear genes cause a reduction in the hybrid fitness (Barton, 1979). These zones will also move as an advancing wave in a homogenous population, but are stabilised in places where there is reduced migration. This can arise either from barriers to migration, in regions of low population density or simply when the boundaries of the population come close together in a narrow corridor. The approach of Barton (1979) has been previously applied to the spatial spread of a single strain of Wolbachia (Turelli and Hoffmann, 1991). This led Hoffmann and Turelli (1997) to verbally argue that multiple strains of Wolbachia were likely to behave in a similar way to hybrid zones, a conclusion which is borne out by our analysis.

Although our results suggest that incompatible Wolbachia can coexist in neighbouring populations, this result critically depends on the absence of any horizontal or paternal transmission. If this occurs, then it may result in double infections within a single female. It is expected that such infections will rapidly spread through both classes of singly infected populations. The perfect association observed between bacterial strains and mitochondrial types within and between populations suggests that horizontal and paternal transmission of Wolbachia may be very rare (Turelli et al, 1992; Rousset and Solignac, 1995; Schulenburg et al, 2002). However, rare paternal transmission has been observed in D. simulans (Hoffmann and Turelli, 1988), and, under certain unusual ecological circumstances, horizontal transmission can be common (Huigens et al, 2000).
The model generates testable predictions as to the migration rates and bacterial characteristics necessary for different CI strains to coexist in adjacent populations. Therefore, it will be of great interest to identify populations of this type and measure the relevant parameters. Of particular interest would be a fuller description of Wolbachia in C. pipiens, with the aim of discovering whether it exhibits the patterns described by our model.

A number of further questions remain to be answered before we can conclude that Wolbachia can act as the significant factor causing speciation (Hurst and Schilthuizen, 1998; Werren, 1998). How long do these infections persist for? Is cytoplasmic incompatibility an effective barrier to the flow of nuclear genes? The true importance of Wolbachia in causing speciation awaits further empirical data on these questions.

\section{References}

Barr AR (1980). Cytoplasmic incompatibility in natural populations of a mosquito, Culex pipiens L. Nature 283: 71-72.

Barton NH (1979). The dynamics of hybrid zones. Heredity 43: 341-359.

Bolker BM, Grenfell BT (1995). Space, persistence and dynamics of measles epidemics. Phil Trans $R$ Soc Lond B 348: 309-320.

Bordenstein SR, O'hara FP, Werren JH (2001). Wolbachia-induced incompatibility precedes other hybrid incompatibilities in Nasonia. Nature 409: 707-710.

Bouchon D, Rigaud T, Juchault P (1998). Evidence for widespread Wolbachia infection in isopod crustaceans: molecular identification and host feminization. Proc $R$ Soc Lond B 265: 1081-1090.

Breeuwer JAJ, Werren JH (1990). Microorganisms associated with chromosome destruction and reproductive isolation between two insect species. Nature 346: 558-560.

Caspari E, Watson G (1959). On the evolutionary importance of cytoplasmic sterility in mosquitoes. Evolution 13: 568-570.

Courchamp F, Clutton-Brock T, Grenfell B (1999). Inverse density dependence and the Allee effect. TREE 14: 405-410.

Coyne JA, Orr HA (1989). Patterns of speciation in Drosophila. Evolution 43: 362-381.

Fine PEM (1978). On the dynamics of symbiote-dependant cytoplasmic incompatibility in Culicine mosquitoes. J Invert Path 30: 10-18.

Hanski I (1983). Coexistence of competitors in patchy environments. Ecology 64: 493-500.

Hanson WD (1966). Effects of partial isolation (distance), migration, and different fitness requirements among environmental pockets upon steady state gene frequencies. Biometrics 22: 453-468.

Hassell MP, Comins H, May RM (1991). Spatial structure and chaos in insect population dynamics. Nature 353: 255-258.

Hoffmann AA, Turelli M (1988). Unidirectional incompatibility in Drosophila simulans: inheritance, geographic variation and fitness effects. Genetics 119: 435-444.

Hoffmann AA, Turelli M (1997). Cytoplasmic incompatability in insects In: O'Neill SL, Hoffman AA, Werren JH (eds) Influential Passengers: Microbes and Invertebrate Reproduction, Oxford University Press: Oxford. pp 42-80.

Huigens ME, Luck RF, Klaassen RHG, Maas M, Timmermans M, Stouthamer R (2000). Infectious parthenogenesis. Nature 405: 178-179.

Hurst GDD, Schilthuizen M (1998). Selfish genetic elements and speciation. Heredity 80: 2-8.

Jeyaprakash A, Hoy MA (2000). Long PCR improves Wolbachia DNA amplification: wsp sequences found in $76 \%$ of sixtythree arthropod species. Insect Mol Biol 9: 393-405. 
Jiggins FM, Bentley Majerus MEN, Hurst GDD (2001). How many species are infected with Wolbachia? Cryptic sex ratio distorters revealed to be common by intensive sampling. Proc $R$ Soc Lond B 268: 1123-1126.

Karlin S, Mcgregor J (1972). Application of method of small parameters to multi-niche population genetic models. Theor Pop Biol 3: 186-209.

Keeling MJ (1999). Spatial models of interacting populations In: McGlade J (ed) Advanced Theoretical Ecology, Blackwell Scientific Publishing: Oxford. pp 64-99.

Laven H (1951). Crossing experiments with Culex strains. Evolution 5: 370-375.

Laven H (1959). Speciation by cytoplasmic incompatibility in the Culex pipiens complex. Cold Spring Harbour Symp Quant Biol 24: 166-173.

Magnin M, Pasteur N, Raymond M (1987). Multiple incompatibilities within populations of Culex pipiens L. in southern France. Genetica 74: 125-130.

Merçot H, Llorente B, Jacques M, Atlan A, Montchamp-Moreau C (1995). Variability within the Seychelles cytoplasmic incompatability system in Drosophila simulans. Genetics 141: 1015-1023.

Montchamp-Moreau C, Ferveur JF, Jacques M (1991). Geographic distribution and inheritance of three cytoplasmic incompatibility types in Drosophila simulans. Genetics 129: 399-407.

O'Neill SL, Giordano R, Colbert AME, Karr TL, Robertson HM (1992). 16S ribosomal-RNA phylogenetic analysis of the bacterial endosymbionts associated with cytoplasmic incompatibility in insects. Proc Natl Acad Sci USA 89: 2699-2702.

O'Neill RJW, Karr TL (1990). Bidirectional incompatibility between conspecific populations of Drosophila simulans. Nature 348: 178-180.

Pacala SW, Levin SA (1996). Biologically generated spatial pattern and the coexistence of competing species In: Tilman D, Kareiva P (eds) Spatial Ecology: The Role of Space in Population Dynamics and Interspecific Interactions, Princeton University Press: Princeton, NJ.

Rousset F, Raymond M, Kjellberg F (1991). Cytoplasmic incompatibilities in the mosquito Culex-Pipiens - how to explain a cytotype polymorphism. J Evol Biol 4: 69-81.

Rousset F, Solignac M (1995). The evolution of single and double Wolbachia symbioses during speciation in the Drosophila simulans complex. Proc Natl Acad Sci USA 92. 6389-6393.

Schofield P (2002). Spatially explicit models of TurelliHoffmann Wolbachia invasive wave fronts. J Theor Biol 215 121-131.
Schulenburg JHGVD, Hurst GDD, Tetzlaff D, Booth GE, Zakharov IA, Majerus MEN (2002). History of infection with different male-killing bacteria in the two-spot ladybird beetle Adalia bipunctata revealed through mitochondrial DNA analysis. Genetics 160: 1075-1086.

Shoemaker DD, Katju V, Jaenike J (1999). Wolbachia and the evolution of reproductive isolation between Drosophilla recens and Drosophila subquinaria. Evolution 53: 1157-1164.

Slatkin M (1974). Competition and regional coexistence. Ecology 55: $128-134$

Telschow AP, Hammerstein P, Werren JH (2002a). The effect of Wolbachia on genetic divergence between populations: mainland-island model. Int Comp Biol 42: 340-351.

Telschow AP, Hammerstein P, Werren JH (2002b). The effect of Wolbachia on genetic divergence between populations: models with two way migration. Am Nat 160: S54-S66.

Tilman D (1994). Competition and biodiversity in spatially structured habitats. Ecology 75: 2-16.

Turelli M, Hoffmann AA (1991). Rapid spread of an inherited incompatibility factor in California Drosophila. Nature 353: 440-442.

Turelli M, Hoffmann AA (1995). Cytoplasmic incompatibility in Drosophila-Simulans - dynamics and parameter estimates from natural-populations. Genetics 140: 1319-1338.

Turelli M, Hoffmann AA, Mckechnie SW (1992). Dynamics of cytoplasmic incompatibility and mtDNA variation in natural Drosophila-Simulans populations. Genetics 132: 713-723.

Wade MJ, Stevens L (1994). The effect of population subdivision on the rate of spread of parasite-mediated cytoplasmic incompatibility. J Theor Biol 167: 81-87.

Weeks AR, Reynolds KT, Hoffmann AA, Mann H (2002). Wolbachia dynamics and host effects: what has (and has not) been demonstrated? Trends Ecol Evol 17: 257-262.

Werren JH (1997). Biology of Wolbachia Annu Rev Entomol 42: 587-609.

Werren JH (1998). Wolbachia and speciation In: Howard DJ, Berlocher SH (eds) Endless Forms: Species and Speciation, Oxford University Press: Oxford. pp 245-260.

Werren JH, Windsor D, Guo LR (1995). Distribution of Wolbachia among neotropical arthropods. Proc $R$ Soc Lond $B$ 262: 197-204.

West SA, Cook JM, Werren JH, Godfray HCJ (1998). Wolbachia in two insect host-parasitoid communities. Mol Ecol 7: 1457-1465.

Yen JH, Barr AR (1971). A new hypothesis of the cause of cytoplasmic incompatibility in Culex pipiens. Nature 232: 657-658. 